\title{
Immunogenic apoptosis in human acute myeloid leukemia (AML): primary human AML cells expose calreticulin and release heat shock protein (HSP) 70 and HSP90 during apoptosis
}

\author{
HANNE FREDLY ${ }^{1}$, ELISABETH ERSVÆR ${ }^{1}$, BJØRN-TORE GJERTSEN ${ }^{1}$ and $\varnothing$ YSTEIN BRUSERUD $^{1,2}$ \\ ${ }^{1}$ Section for Hematology, Institute of Internal Medicine, University of Bergen; \\ ${ }^{2}$ Section for Hematology, Department of Medicine, Haukeland University Hospital, Bergen, Norway
}

Received December 10, 2010; Accepted January 21, 2011

DOI: 10.3892/or.2011.1229

\begin{abstract}
Several previous studies have demonstrated that both conventional cytotoxic drugs as well as targeted therapeutics can induce apoptosis in primary human acute myelogenous leukemia (AML) cells. However, the apoptotic phenotype of dying AML cells has been less extensively characterized. Even though specific antileukemic immune reactivity is important in AML, especially for allotransplanted patients, it has not been investigated whether dying primary human AML cells show phenotypic characteristics consistent with immunogenic apoptosis [calreticulin exposure, heat shock protein (HSP) release]. We therefore investigated whether in vitro cultured primary human acute myeloid leukemia (AML) cells show calreticulin exposure and HSP70/HSP90 release during spontaneous (stress-induced) apoptosis when cultured in medium alone and when cultured in the presence of antileukemic drugs. Both surface exposure of calreticulin and release of HSP70 and HSP90 was detected but showed a wide variation between patients. This variation was also maintained when the AML cells were cultured in the presence of cytotoxic drugs (cytarabine, daunorubicin, mitomycin), all-trans retinoic acid (ATRA) and valproic acid. Finally, AML cells collected during in vivo ATRA therapy showed increased calreticulin exposure during spontaneous in vitro apoptosis, suggesting that in vivo pharmacotherapy can modulate the apoptotic phenotype. To conclude, apoptotic AML cells can show phenotypic characteristics consistent with immunogenic apoptosis, but there is a wide variation between patients and the level of calreticulin exposure/HSP release seems to depend on individual patient characteristics rather than the apoptosisinducing agent.
\end{abstract}

Correspondence to: Dr Øystein Bruserud, Department of Medicine, Haukeland University Hospital, N-5021 Bergen, Norway E-mail: oystein.bruserud@haukeland.no

Key words: acute myeloid leukemia, apoptosis, calreticulin, heat shock protein 70 , heat shock protein 90

\section{Introduction}

Acute myelogenous leukemia (AML) is an aggressive malignancy characterized by accumulation of immature leukemia cells in the bone marrow (1). The median age at diagnosis is 60-70 years, and the most intensive and potentially curative therapy is usually possible only for otherwise healthy patients below 60-70 years of age (1). The drugs used in clinical treatment induce apoptotic cell death and thereby hematological remission $(2,3)$. However, recent studies indicate that antileukemic immune reactivity is also important for long-term AML-free survival; this is suggested by the increased survival for patients with early lymphoid reconstitution after both conventional chemotherapy and stem cell transplantation (4-8).

Apoptosis was originally thought to be immunologically silent, but recent results distinguish between immunogenic and non-immunogenic apoptosis (9-11). Immunogenic apoptosis is characterized by cell surface exposure of calreticulin (11) and extracellular release of heat shock protein (HSP) 70 and HSP90 (12). Calreticulin is a chaperone normally localized to the endoplasmatic reticulum (13), and its exposure induces phagocytosis by antigen-presenting dendritic cells and later development of antigen-specific $\mathrm{T}$ cell reactivity $(9,10)$. However, at least in experimental models the exposure of calreticulin depends on the apoptosis inducer, e.g. it is seen after exposure to anthracyclins but not mitomycin $(9,11)$.

Induction of apoptosis in AML cells is regarded as an important effect of both conventional chemotherapy as well as the new targeted therapeutic strategies that are considered for AML treatment (14-16). Previous studies have then focused on induction of apoptosis in the AML cells, whereas the apoptotic phenotype has not been characterized in detail even though antileukemic immune reactivity has a documented clinical effect for these patients especially after allogeneic stem cell transplantation (17). To the best of our knowledge only one study has examined immunogenic characteristics in primary human AML cells; this was a small study including only 4 patients and the results suggest that calreticulin can be exposed by AML cells for certain patients (18). We have therefore characterized calreticulin exposure together with the release of HSP70 and HSP90 by apoptotic primary human AML cells for a large group of patients. 


\section{Materials and methods}

Primary human AML cells. Primary human AML cells were collected after informed consent from 37 patients with untreated disease (Table I). In addition, we also studied 7 patients included in a previous study and treated with all-trans retinoic acid (ATRA) plus valproic acid and theophylline $(19,20)$; we then compared in vitro calreticulin exposure by cells harvested before therapy, after two days of treatment with ATRA alone and after five additional days with ATRA plus valproic acid and theophyllin. All patients had high peripheral blood leukemic cell counts, and enriched AML cells could therefore be prepared from peripheral blood samples by density gradient separation alone (21). Cells were cryopreserved and stored in liquid nitrogen until used (22). Light microscopy showed that AML blasts constituted at least $95 \%$ of the cells, the major contamination being small lymphocytes.

Cell culture. Reagents. Cells were cultured in StemSpan serum-free medium (Stem Cell Technologies Inc., Vancouver, $\mathrm{BC}$, Canada) supplemented with $100 \mu \mathrm{g} / \mathrm{ml}$ of gentamicin (Schering-Plough AG, Berlin, Germany) (23). Valproic acid (Destin $\mathrm{GmbH}$, Hamburg, Germany) was used at 600 and $2400 \mu \mathrm{M} ; 600 \mu \mathrm{M}$ increases AML cell proliferation for a subset of patients whereas decreased proliferation is generally observed for $2400 \mu \mathrm{M}$ (24). ATRA (Roche, Oslo, Norway) was used at $1.1 \mu \mathrm{M}(24)$. Unless otherwise stated mitomycin (Medac $\mathrm{GmbH}$, Wedel, Germany) was used at $25 \mu \mathrm{M}$, daunorubicin (Sanofi-Aventis, Lysaker, Norway) at 2 and $4 \mu \mathrm{M}$ and cytarabine (Pfizer, Lysaker, Norway) at 1, 10 and $100 \mathrm{nM}$. Recombinant human Flt3-ligand (Flt3L), stem cell factor (SCF) and granulocyte-macrophage colony-stimulating factor (GM-CSF) were purchased from Peprotech Inc (Rocky Hill, NJ, USA) and used at $20 \mathrm{ng} / \mathrm{ml}$.

Analysis of AML cell apoptosis and HSP levels. Cells were thawed for each experiment (22) and cultured at $1 \times 10^{6}$ cells $/ \mathrm{ml}$ in 24-well tissue culture plates (Nunc AS, Roskilde, Denmark) at $37^{\circ} \mathrm{C}$ in a humidified atmosphere of $5 \% \mathrm{CO}_{2}$ for $4 \mathrm{~h}$ unless otherwise stated before i) cells were harvested for flow cytometric analyses; or ii) supernatants were analysed for HSP70/HSP90 levels by ELISA analyses (Assay Designs, Inc., Michigan, USA). Intracellular HSP levels were analysed for $2 \times 10^{6}$ AML cells in $1 \mathrm{ml}$ lysis solution and analyzed by Multiplex HSP/chaperon 8-plex MultiBead Kit for flow cytometry (Stressgen; Victoria, BC, Canada).

${ }^{3} \mathrm{H}$-thymidine incorporation assay. As described in detail previously $(25,26)$, AML cells were cultured in medium alone or medium containing Flt3L+SCF+GM-CSF $\left(5 \times 10^{4}\right.$ cells/well, $150 \mathrm{ml}$ medium per well) in flat-bottomed 96-well microtiter plates (Nucleon ${ }^{\mathrm{TM}} \Delta$ Surface; Nunc Roskilde, Denmark). After six days $\left[{ }^{3} \mathrm{H}\right]$-thymidine $(37 \mathrm{kBq} /$ well; TRA 310 , Amersham International, Amersham, UK) was added in $20 \mathrm{ml}$ saline and nuclear radioactivity assayed $18 \mathrm{~h}$ later. The median counts per minute (cpm) of triplicate cultures were used for analysis of the results. Negative controls were included in each experiment and corresponded to $<200 \mathrm{cpm}$.

Flow cytometric analysis of AML cell viability and calreticulin exposure. Reagents. FITC-conjugated anti-Annexin $\mathrm{V}$ was obtained from NeXins Research (Kattendijke, The
Table I. Clinical and biological characteristics of 37 AML patients included in the study.

Age; median (range)

66 years $(24-88)$

Gender (female/male)

$17 / 20$

FAB classification (number of patients)

$\mathrm{M} 0 / \mathrm{M} 1$

$3 / 11$

$\mathrm{M} 2$

8

M4/M5

$10 / 5$

Cytogenetic abnormalities ${ }^{\mathrm{a}}$

Normal

Intermediate

3

Good

2

Adverse

Flt3-internal tandem duplication ${ }^{\mathrm{a}}$

NPM-1 mutation

6

${ }^{a}$ Cytogenetic analysis was available for 31 patients and they were classified according to the MRC guidelines (36). Flt3 mutations were analysed in 29 patients and NPM-1 mutations in 22 patients.

Netherlands). PacBlu-conjugated anti-Annexin V, live/dead fixable violet dead cell stain kit and Alexa Fluor 647-conjugated, goat anti-rabbit IgG was obtained from Invitrogen (Paisley, UK). Polyclonal rabbit anti-calreticulin was purchased by Cell Signaling Technology (Danvers, MA, USA), and FITCconjugated mouse monoclonal anti-human CD3, anti-human CD5 and PeCy7-conjugated mouse anti-human CD5 by BD Bioscience Pharmingen (San Jose, CA, USA). Phosphatebuffered saline (PBS) was delivered by Farma Holding (Oslo, Norway), bovine serum albumin (BSA) by Roche, human immunoglobulin by Octapharma AG (Lachen, Switzerland) and paraformaldehyde (PFA) by Electron Microscopy Sciences (Hatfield, PA, USA).

Staining of AML cells. Cells were washed once in ice-cold PBS followed by staining as recommended for the dead/live fixable violet dead cell stain kit, including FITC-conjugated anti-Annexin V. Samples were thereafter washed once, fixed for $15 \mathrm{~min}$ in $0.5 \% \mathrm{PFA} / \mathrm{PBS}$, then washed once in $2 \% \mathrm{BSA} /$ PBS and blocked with human immunoglobulin $0.2 \mathrm{mg} / \mathrm{ml}$. Staining with monoclonal antibodies was finally performed on ice with rabbit anti-calreticulin antibodies and anti-CD3 or anti-CD5 for $30 \mathrm{~min}$. The samples were thereafter washed once and incubated on ice for 30 min with Alexa 647-conjugated goat anti-rabbit IgG.

Flow cytometry analyses. Flow cytometry was performed by Facs Canto 2 (BD Bioscience Pharmingen) and samples were analysed using the Flow-Jo software (Tree Star, Ashland, OR, USA). Forward scatter (FSC) and side scatter (SSC) analysis was used to identify intact cells, and by using SSC combined with $\mathrm{CD} 3 / \mathrm{CD} 5$ expression the lymphocyte population could be separated from the AML cells. This analysis verified that our gradient-separated AML cell populations included at least 95\% AML cells. The identified leukemia cells were also analysed by FSC/SSC to identify viable and dead cells. However, AML patients are heterogeneous with 


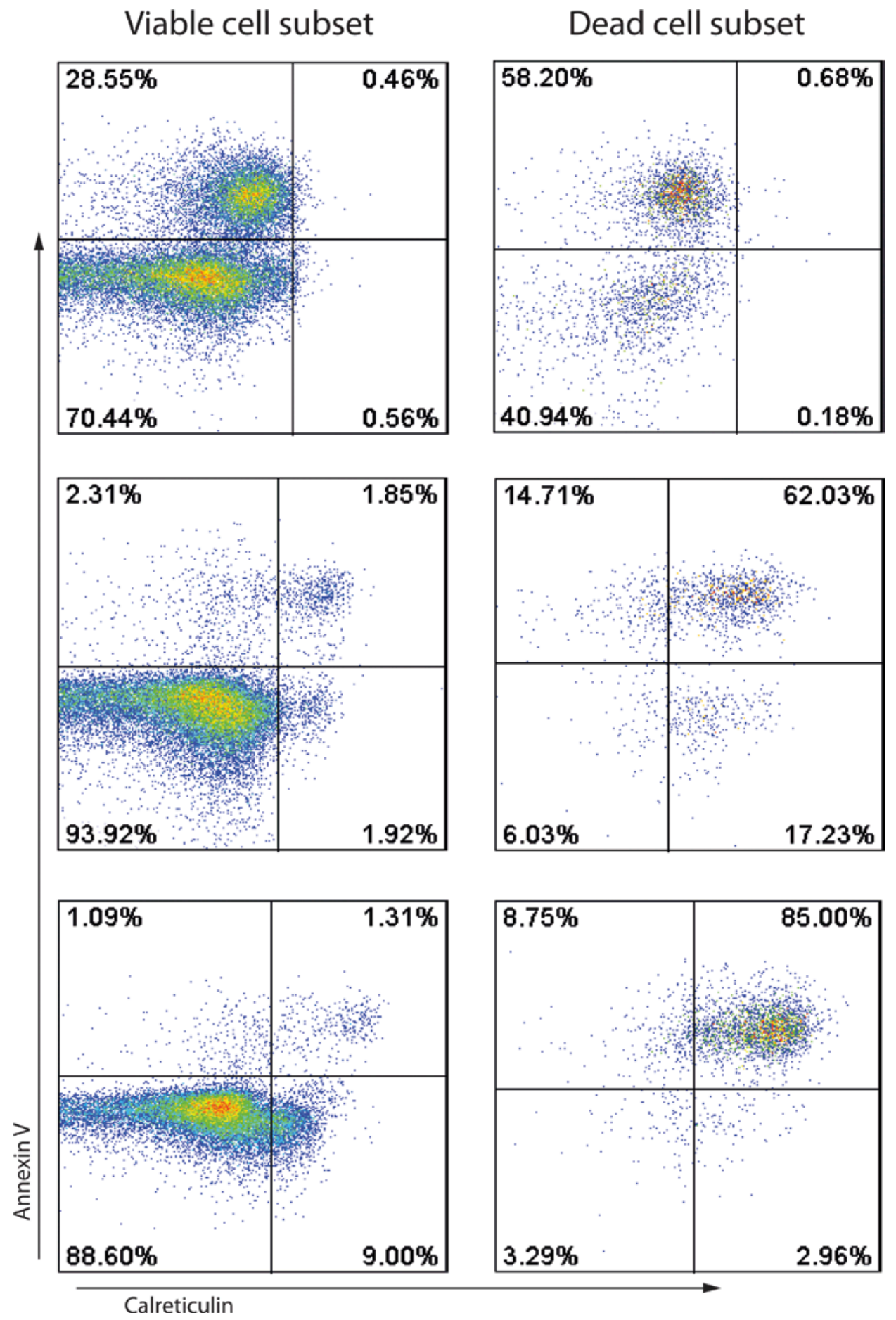

Figure 1. Expression of Annexin V and calreticulin by viable (dye-excluding) and dead (dye-permeable) primary AML cells derived from 3 patients (upper/ middle/lower). Cryopreserved AML cells were cultured for $4 \mathrm{~h}$ before staining and flow cytometric analysis. The cells were gated as described in the text, and the viable (left) and dead (right) subsets were analysed separately for Annexin V (y-axis) and calreticulin (x-axis) expression. The percentage of cells within each of the four subset in the separate analyses is given in the figures.

regard to leukemic cell morphology, and for certain patients it was difficult to identify the two cell subsets by FSC/SSC analysis alone. The dead/live viability/vitality analysis was therefore used to verify this gating. Finally, the expression of Annexin $\mathrm{V}$ and calreticulin was analysed separately for the dead and viable AML cell subsets. As expected from previous studies we could identify distinct Annexin V-positive and -negative cell subpopulations (27). Calreticulin-positive cells could then be identified as separate populations among viable or dead cell subsets. This discrimination is illustrated by the results for three representative patients presented in Fig. 1, and the calreticulin-positive cell populations were also classified as positive when using a threshold for positivity corresponding to the fluorescence intensity level at which $0.5 \%$ of the cells in the Ig-blocked controls were classified as positive after staining with only the secondary antibody. 
Statistical analysis. The Wilcoxon's test for paired samples and the Mann-Whitney test were used for statistical comparisons and differences regarded as significant when $\mathrm{p}<0.05$. For correlation analysis the Kendall tau rank correlation coefficient was used.

\section{Results}

Standardization of the experimental in vitro models. We used an experimental in vitro model with culture of primary human AML cells in serum-free medium for analysis of apoptosis; this model has been characterized in detail previously $(23,27)$. Cytarabine was then tested at three different concentrations that were selected based on their effects on cytokine-dependent (Flt3L+SCF+GM-CSF) AML cell proliferation. Cells derived from 19 unselected patients were then investigated in the ${ }^{3} \mathrm{H}$-thymidine incorporation assay, the median proliferation in drug-free controls then corresponded to $3998 \mathrm{cpm}$ (range $<1000-66,161 \mathrm{cpm}$ ) and cytarabine was tested at ten-fold dilutions $(10 \mu \mathrm{M}-0.1 \mathrm{nM})$. We selected three different cytarabine concentrations that caused either i) a weak inhibition corresponding to $<20 \%(1 \mathrm{nM})$, ii) approximately $50 \%$ inhibition $10 \mathrm{nM}$ ) and iii) an inhibition corresponding to the plateau of maximal (i.e. $>80 \%)$ inhibition $(100 \mathrm{nM}$ ).

We compared apoptosis induction and calreticulin expression for 4 unselected patients after $18 \mathrm{~h}$ of culture when using daunorubicin 1, 2, 4 and $6 \mu \mathrm{M}$ and mitomycin 15 and $25 \mu \mathrm{M}$. Daunorubicin 2 and $4 \mu \mathrm{M}$ caused higher levels of calreticulin exposure and were therefore used in the present experiments. The two mitomycin concentrations were comparable with regard to cell viability and calreticulin-exposing cells, and $25 \mu \mathrm{M}$ was used in the following experiments. These drug concentrations have also been used in previous studies $(11,28)$.

Primary AML cells will undergo spontaneous apoptosis during in vitro culture; the degree of apoptosis then shows a wide variation between patients (27). In all our studies we used cryopreserved cells that showed a high viability generally exceeding $80 \%$ at initiation of culture. In pilot experiments AML cells derived from 4 unselected patients were cultured with and without the drugs for 2, 4, 6, 7 and $18 \mathrm{~h}$. A gradual decrease in the fraction of viable cells was then seen for all drugs, especially for all the cytotoxic drug cultures that showed a large majority of dead cells after $18 \mathrm{~h}$. As an example, for daunorubicin $2 \mu \mathrm{M}$ the median level of dead (dye-permeable) cells was not altered after $2 \mathrm{~h}$ (9.9\% dead cells) but thereafter showed a gradual increase when comparing $4 \mathrm{~h}$ (median 14.7\%), $6 \mathrm{~h} \mathrm{(23.4 \% ),} 7 \mathrm{~h} \mathrm{(23.3 \% )}$ and $18 \mathrm{~h} \mathrm{(82.9 \% )} \mathrm{of} \mathrm{incu-}$ bation. After 4-7 h a distinct population of dead cells was identified for all patients and drugs, a marked variation between different patients could be demonstrated, and the percentages of calreticulin-exposing cells was comparable during the whole time period. Calreticulin exposure is an early event during apoptosis (10) and we therefore investigated its expression after $4 \mathrm{~h}$ of culture in the following experiments.

Calreticulin is exposed by apoptotic primary human AML cells for most patients. Primary human AML cells derived from 37 patients (Table I) were incubated in medium alone for $4 \mathrm{~h}$ before analysis of Annexin $\mathrm{V}$ and calreticulin exposure by the viable (dye-excluding) and non-viable (dye-permeable)

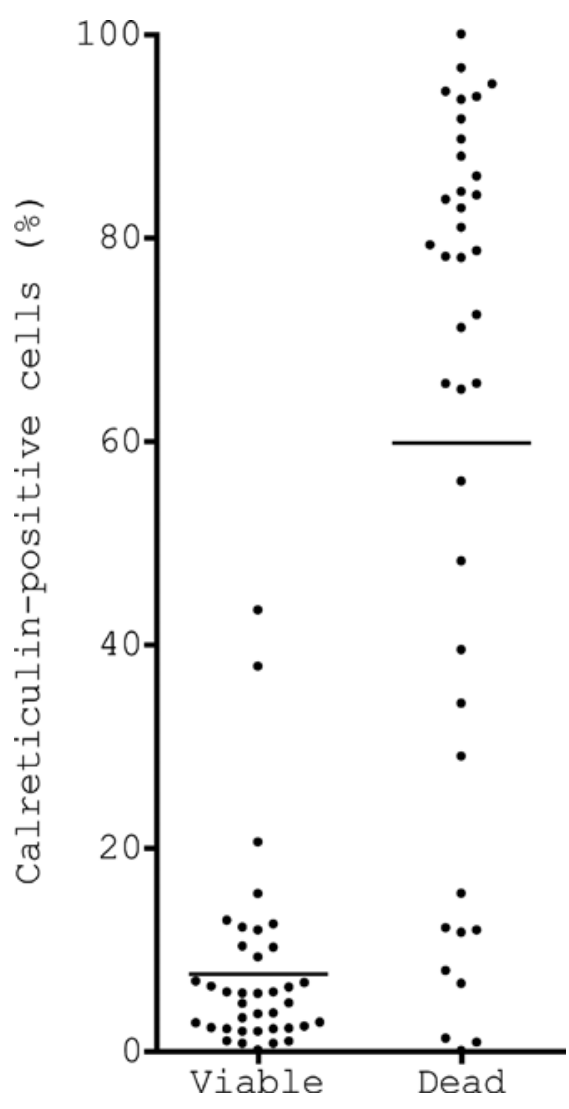

Figure 2. Calreticulin exposure by primary human AML cells after $4 \mathrm{~h}$ of in vitro incubation. Leukemic cells derived from 37 unselected patients were examined, and the total number of calreticulin-positive cells among viable (dye-excluding) and dead (dye-permeable) AML cell subsets were analysed by flow cytometry. The results are presented as the percentage of positive cells among the total viable or dead cell subsets. The mean level for each subset is indicated in the figure.

cell subsets. Firstly, most viable cells were Annexin V-calreticulin- (mean level 77.0\%), but a low percentage of calreticulin ${ }^{+}$ cells was also detected for most patients (Fig. 2; median $4.7 \%$ of total viable cells, range $0.1-43.4 \%$ ) and these cells were both Annexin $\mathrm{V}^{-}$calreticulin ${ }^{+}$(median 1.8\%, range $0.1-14.8 \%$ ) and $\mathrm{AnnexinV}^{+}$calreticulin ${ }^{+}$(median 1.9\%, range 0.00-32.4\%). Secondly, for the dead (dye-permeable) cell subset the percentage of calreticulin ${ }^{+}$cells showed a wider variation between patients. When comparing the overall results the percentage of calreticulin-positive cells was significantly higher for the non-viable (Fig. 2; median level 72.4\%, $\mathrm{p}<0.0001)$ than for the corresponding viable cell subsets. It is unlikely that this increased calreticulin staining is due to non-specific antibody permeability, because incubation of cells with a non-specific fluorescein-conjugated antibody was included in all our experiments and no evidence of staining was observed in these control samples (data not shown). Most calreticulin ${ }^{+}$cells among the nonviable cells were Annexin $\mathrm{V}^{+}$(median 65.7\%) with Annexin $\mathrm{V}^{-}$calreticulin ${ }^{+}$cells usually being a minor subset (median 0.2\%); this is also different from the viable cells. Finally, the percentage of calreticulin-positive cells showed no correlation with FAB classification, genetic abnormalities or expression of the CD34 stem cell marker (data not shown). 


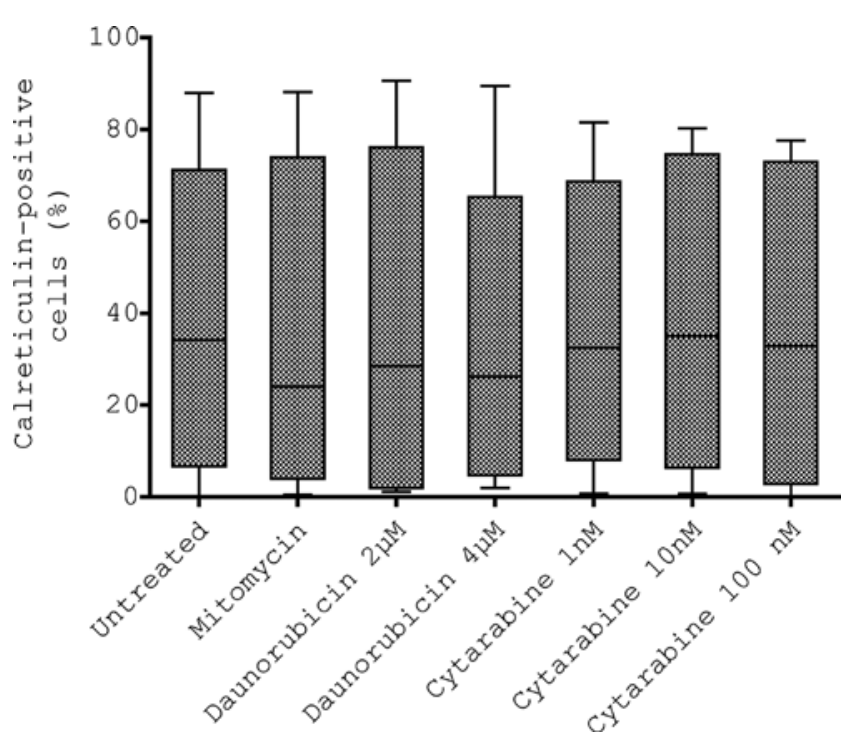

Figure 3 . The percentage of calreticulin-positive cells among primary human AML cells cultured in medium alone or in the presence of cytotoxic drugs. Leukemic cells were derived from 15 unselected patients and cultured for $4 \mathrm{~h}$ in vitro before the percentage of calreticulin-positive cells were determined by flow cytometry for the viable and non-viable AML cell subsets. The results for the non-viable cell subsets are presented as box plots with the sample minimum value, the lower quartile, median value, upper quartile and sample maximum value for cells cultured in medium alone (untreated) and in the presence of mitomycin, daunorubicin ( 2 and $4 \mu \mathrm{M})$ and cytarabine $(0.1,1$ and $10 \mathrm{nM})$

The variation in calreticulin exposure is maintained after incubation with cytotoxic drugs. The effects of various drugs on viability and calreticulin exposure were studied more in detail for an unselected subset of 15 patients. The mean percentage of viable leukemic cells after $4 \mathrm{~h}$ of incubation corresponded to $75.5 \%$ for the drug-free controls. A small but statistically significant reduction of the viability was observed only for daunorubcin $4 \mu \mathrm{M}$ (mean viability $70.4 \%, \mathrm{p}=0.01$ ), but there was still a statistically significant correlation between the viability in these cultures and the corresponding drug-free controls $(r=0,823, p<0.01)$. In contrast, the viability was not significantly altered by valproic acid (mean viability $77.5 \%$ for $600 \mu \mathrm{M}$ and $77.1 \%$ for $2400 \mu \mathrm{M}$ ), cytarabine (e.g. mean $73.5 \%$ for $100 \mathrm{nM}$ ), daunorubicin $2 \mu \mathrm{M}$ (mean $73.6 \%$ ), ATRA (mean $72.8 \%$ ) and mitomycin $25 \mu \mathrm{M}$ (mean $71.7 \%$ ).

The calreticulin exposure of the AML cells derived from these 15 patients was also compared for cultures prepared in medium alone or with cytarabine (1, 10 and $100 \mathrm{nM})$, daunorubicin $(2$ and $4 \mu \mathrm{M})$ and mitomycin $(25 \mu \mathrm{M})$. The same AML cell subsets were detected as in the corresponding drug-free controls, and the presence of the cytotoxic drugs did not cause any statistically significant alteration in the percentage of calreticulin-exposing cells (Fig. 3); the percentages remained low for the viable (dye-excluding) cell subset, and for the non-viable subset the variation between patients was maintained and significant correlations were observed between the percentages of calreticulin-exposing cells in control cultures and cultures containing mitomycin $(r=0.67$, $\mathrm{p}<0.01$ ), daunorubicin (e.g. $4 \mu \mathrm{M}, \mathrm{r}=0.619, \mathrm{p}<0.01$ ) and cytarabine (e.g. $10 \mathrm{nM}, \mathrm{r}=0.802$, $\mathrm{p}<0.01$ ). Thus, primary human
AML cells can express calreticulin on their cell surface, but there is a wide variation between patients especially for non-viable cells, and this variation is maintained also after incubation with cytotoxic drugs. Similar results were even seen when calreticulin-exposure was compared separately for Annexin V-positive and -negative cells (data not shown). The results for drug-free controls and all drug-containing cultures were also reproduced in independent experiments for three of the patients (data not shown).

Differences in calreticulin exposure are maintained both after in vitro and in vivo exposure of primary human AML cells to ATRA or valproic acid. We compared calreticulin exposure for primary human AML cells cultured in medium alone and in the presence of either valproic acid (600 and $2400 \mathrm{nM}$ ) or ATRA. When analyzing the overall results no statistically significant effects on calreticulin exposure were observed for any of these two drugs. The same AML cell subsets were identified for drug-containing cultures and drug-free controls, and there were significant correlations between the percentages of calreticulin-positive cells among non-viable cells in control cultures and corresponding cultures containing valproic acid $600 \mathrm{nM}(\mathrm{r}=0.714, \mathrm{p}=<0.01)$, valproic acid $2400 \mathrm{nM}(\mathrm{r}=0.695, \mathrm{p}<0.01)$ and ATRA $(\mathrm{r}=0.771, \mathrm{p}<0.01)$. Finally, valproic acid and ATRA did not have any significant effects on calreticulin exposure in the presence of cytarabine $(10 \mathrm{nM})$ and daunorubicin $(4 \mu \mathrm{M})$ either (data not shown).

We compared spontaneous in vitro apoptosis for AML cells collected from 7 patients before therapy, after in vivo treatment with ATRA alone for 2 days and after 5 additional days with ATRA combined with valproic acid and theophyllin. There was a small but significant increase in calreticulinexposure by the dye-excluding AML cell subset after two days of in vivo ATRA therapy (median level of calreticulin-positive cells $9.8 \%$, range 5.1-68.1\%) compared with cells collected before treatment (median level 5.7\%, range 3.2-65.8, $\mathrm{p}=0.02$ ), but otherwise the in vitro calreticulin exposure did not differ between cells collected before and during treatment (data not shown). Thus, patient-dependent characteristics are the most important determinants for calreticulin exposure both after in vivo and in vitro exposure to these drugs.

In vitro-cultured AML cells show spontaneous release of HSP70 and HSP90. Immunogenic apoptosis is characterized by the release of HSP70 and HSP90 in addition to calreticulin exposure $(9,12)$. We therefore investigated the spontaneous release of HSP70 and HSP90 for in vitro-cultured primary human AML cells. First we compared the HSP70/HSP90 release when leukemic cells derived from 14 unselected patients were cultured for 4 and $18 \mathrm{~h}$ in medium alone. A wide variation in HSP levels was observed between individual patients. The HSP70 levels were relatively low after $4 \mathrm{~h}$ and showed a significant increase after $18 \mathrm{~h}$ of culture (Fig. 4A, $\mathrm{p}=0.001$ ), whereas the HSP90 levels did not differ significantly after 4 and $18 \mathrm{~h}$. Furthermore, HSP70 and HSP90 levels after $18 \mathrm{~h}$ showed a significant correlation (a total of 18 patients examined, $\mathrm{r}=0.515, \mathrm{p}=0.003$ ) whereas the 4-h levels did not (a total of 25 patients examined, see below).

We investigated the spontaneous release of HSP70 and HSP90 after $4 \mathrm{~h}$ of in vitro culture for a total of 25 patients. 
A

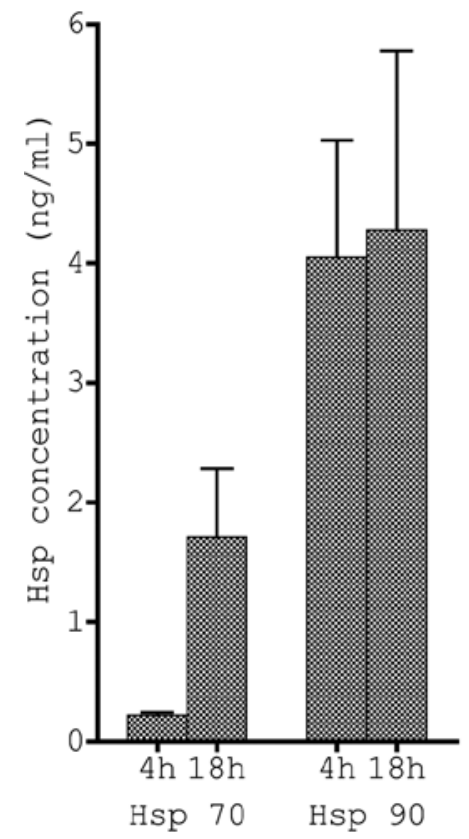

B

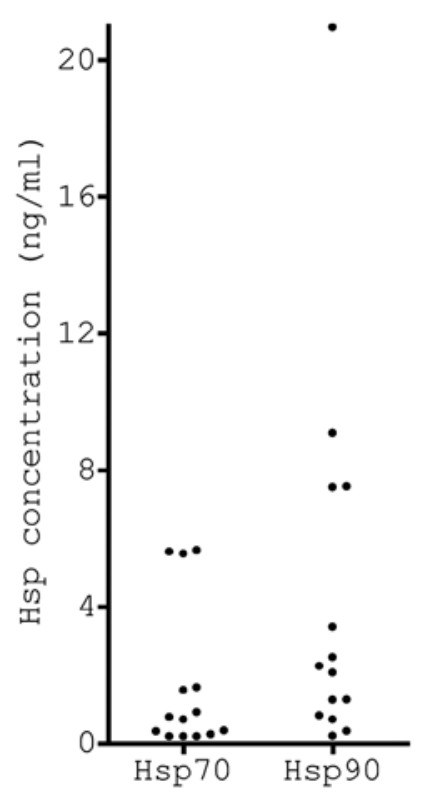

C

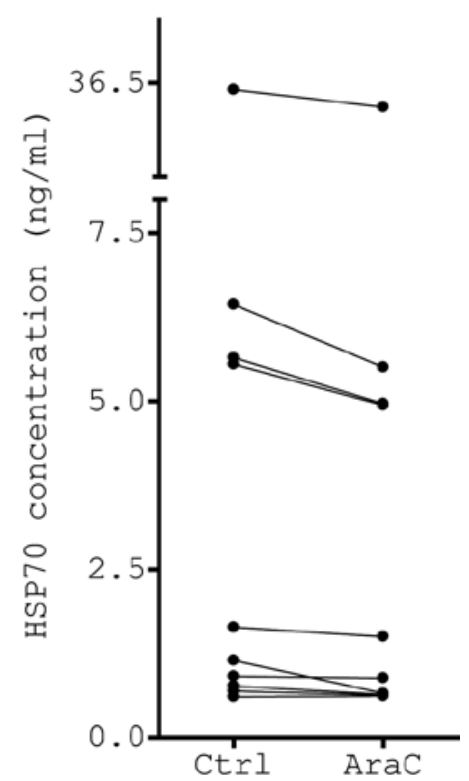

Figure 4. Release of HSP70 and HSP90 by in vitro-cultured primary human AML cells. (A) Leukemic cells were derived from 14 unselected patients and cultured for 4 and $18 \mathrm{~h}$ before HSP levels were analysed in the supernatants. (B) The levels of HSP70 and HSP90 are compared for 14 patients when AML cells derived from 14 unselected patients were cultured for $4 \mathrm{~h}$ before HSP levels were determined; the figure presents the levels for each individual patient. (C) Leukemic cells from 10 unselected patients were cultured for $18 \mathrm{~h}$ in medium alone (Ctrl) and with cytarabine (AraC) $10 \mathrm{nM}$, and the HSP70 levels in cytarabine-containing cultures were then significantly decreased (Wilcoxon's test for paired samples, $\mathrm{p}=0.004$ ).

The release of both HSP70 and HSP90 showed a wide variation between patients (Fig. 4B) with no significant correlation with morphological differentiation (i.e. FAB classification) or genetic abnormalities. Neither HSP70 nor HSP90 levels showed any significant correlation with calreticulin exposure of the AML cells. Finally, the HSP levels showed no correlations with the percentage of viable AML cells or the intracellular levels of HSP70/90 (data not shown).

Pharmacological modulation of HSP70/HSP90 release by primary AML cells. We investigated HSP70/HSP90 levels in supernatants when primary human AML cells derived from 6 unselected patients were cultured for $4 \mathrm{~h}$ either in medium alone or in the presence of daunorubicin $4 \mu \mathrm{M}$, mitomycin $25 \mu \mathrm{M}$, cytarabine $10 \mathrm{nM}$ and valproic acid $600 \mathrm{nM}$. The analysis of the control cultures confirmed the wide variation between patients in HSP release, a similar wide variation was observed in the presence of all the drugs and none of the drugs had any significant effect on the HSP levels when the overall results were analysed (data not shown).

We investigated HSP70/90 levels when AML cells derived from 10 unselected patients were cultured for $18 \mathrm{~h}$ in medium alone or in the presence of daunorubicin $4 \mu \mathrm{M}$, mitomycin $25 \mu \mathrm{M}$, cytarabine $10 \mathrm{nM}$ and valproic acid $600 \mathrm{nM}$. Again the analysis of the control cultures confirmed that there was a wide variation between individual patients in the release of both HSP70 and HSP90. Only cytarabine $10 \mathrm{nM}$ had a minor but statistically significant effect and reduced HSP70 levels compared with control cultures (Fig. 4B, p=0.004), but the wide variation in HSP levels between individual patients was maintained and a significant correlation with control levels was observed for HSP90 ( $\mathrm{r}=0.911$, $\mathrm{p}<0.01)$ and even for HSP70 $(r=0.956, p<0.01)$ in the presence of cytarabine. Finally, the wide variation between patients was also maintained in the presence of the other drugs, and significant correlations were detected between HSP70/HSP90 levels in drug-free controls versus cultures containing daunorubicin (HSP70: $\mathrm{r}=0.644, \mathrm{p}<0.01$; HSP90: $\mathrm{r}=1.00, \mathrm{p}<0.01$ ), mitomycin (HSP70: $\mathrm{r}=0.822, \mathrm{p}<0.01$; HSP90: $\mathrm{r}=0.911, \mathrm{p}<0.01)$ and valproic acid (HSP70: $\mathrm{r}=0.822, \mathrm{p}<0.01$; HSP90 r=0.956, $\mathrm{p}<0.01$ ). Finally we compared HSP70/HSP90 release for AML cells collected before and during in vivo treatment with ATRA, valproic acid and theophyllin. The in vitro HSP release did not differ between cells collected before and during this treatment (data not shown).

\section{Discussion}

Antileukemic T cell reactivity is important for the AML-free survival after allogeneic stem cell transplantation $(4,7,29)$ and probably also after conventional chemotherapy and autotransplantation $(5,8)$. Furthermore, the levels of circulating AML-recognizing $\mathrm{T}$ cells can increase after chemotherapy (17). Induction of immunogenic apoptosis with calreticulin exposure and HSP release by the AML cells may then be important, and in the present study we describe that dying primary human AML cells can expose calreticulin and release HSP70 and HSP90.

We included only patients with relatively high peripheral blood blast counts, and highly enriched AML cell popula- 
tions could then be prepared by density gradient separation alone without the risk of inducing functional alterations in the AML cells by more extensive cell separation procedures [for a detailed discussion and additional references see (21)]. Although the included patients are representative for AML patients in general with regard to the most important prognostic parameters (25), our results should be interpreted with care and may be representative only for the subset of patients with relatively high levels of circulating AML cells.

The cell populations showed high viability generally exceeding $70 \%$ after cryopreservation, but the viability decreased during culture due to spontaneous or stress-induced in vitro apoptosis (27). The degree of spontaneous apoptosis differs between patients and depends on intracellular levels of HSP70 and HSP90 and the BCL2:BAX ratio (27). Experimental models based on in vitro culture of cryopreserved primary human AML cells thus cannot avoid a background of stress-induced spontaneous apoptosis even when using optimal and highly standardized culture conditions as described in our methodological publications $(21,25,27)$. However, in our opinion the spontaneous apoptosis reflects biological differences of clinical relevance because HSP-levels as well as the BCL2:BAX ratio seem to have prognostic impact in AML $(30,31)$. As expected stress-induced apoptosis due to the ex vivo handling of the AML cells was also observed in our present study, and the apoptosis in drug-containing cultures represents additional pharmacological modulation on a background of stress-induced cell death. However, the advantages of using cryopreserved instead of freshly prepared AML cells are several: i) reproducibility can be documented; ii) detailed biological characterization of the cells from additional follow-up experiments is available, and iii) follow-up studies are possible.

As discussed in detail previously (25), we only included patients with high peripheral blood blast counts in our studies. The majority of contaminating cells among AML blasts were small lymphocytes, and the flow cytometric analysis verified the high purity and excluded the lymphocytes from the cell death analyses. Previous studies of stress-induced in vitro apoptosis as well as our present studies of primary AML cells were based on the same standardized in vitro culture conditions and flow cytometric analyses $(27,32,33)$. In the previous studies the following cell populations were identified: i) a relatively large population of viable Annnexin $V^{-}$dyeexcluding cells; ii) another population of dead Annexin $\mathrm{V}^{+}$ dye-permeable cells; iii) a small population of early apoptotic Annexin $\mathrm{V}^{+}$dye-excluding cells; and iv) a population of dead dye-permeable Annexin $\mathrm{V}^{-}$cells. Similar populations could be identified in our present study (Fig. 1).

Our decision to analyse calreticulin exposure in primary human AML cells after $4 \mathrm{~h}$ of culture was based on previous experimental studies and our own pilot experiments. Firstly, calreticulin exposure is an early apoptotic event (10) and we therefore analysed the cells when we had only a small population of dead cells. Secondly, our live-dead assay is based on dye permeability that is present for necrotic cells in our standardized in vitro model (27). Our studies have documented that the early events detected during these first $4 \mathrm{~h}$ represent the initial steps leading to gradually increasing apoptosis in drug-free and especially in drug-containing cultures $(19,27)$. Thirdly, our initial kinetic analyses $(2,4,6$, 7 and $18 \mathrm{~h}$ of culture) showed that the viability after $18 \mathrm{~h}$ was below $20 \%$ for all drug-containing cultures, but a difference in AML cell viability between drug-free controls and drugcontaining cultures was detectable already after 4-7 h. There was no major increase in calreticulin exposure during this treatment. For these reasons we investigated the pharmacological effects on calreticulin translocation after $4 \mathrm{~h}$ of in vitro drug exposure.

When using standardized in vitro models we observed that primary human AML cells could expose calreticulin and release HSP70 and HSP90 during spontaneous or stress-induced in vitro apoptosis. However, there was a wide variation between patients with regard to all three parameters, an observation showing that calreticulin exposure and HSP release depend on biological characteristics that differ between patients. This variation was maintained when the AML cells were exposed to cytotoxic (daunorubicin, cytarabine, mitomycin) or noncytotoxic antileukemic drugs (ATRA, valproic acid) in vitro. Previous experimental studies in animal models have demonstrated that daunorubicin can induce immunogenic apoptosis in cancer cells and thereby initiate an anticancer $\mathrm{T}$ cell response, whereas this is not seen for mitomycin (9). However, our present observations suggest that the individual differences between patients are more important than exposure to various antileukemic drugs with regard to these immunostimulatory characteristics. Cytarabine is not an exception to this; even though this drug altered the HSP70 levels the wide variation between patients was still maintained.

It should be emphasized that calreticulin exposure was observed both for Annexin $\mathrm{V}^{-}$and Annexin $\mathrm{V}^{+}$dye-excluding cells as well as for dye-permeable cells. These observations suggest that calreticulin exposure is an early event during apoptosis. However, high calreticulin exposure was observed especially for the dye-permeable cells; the strong staining of the dye-permeable cells probably represents true exposure and not staining of intracellular calreticulin because i) control cultures showed no evidence for non-specific permeability to fluorochrome-conjugated antibodies; iii) previous studies have also demonstrated that an additional permeabilization step is needed for intracellular staining even in non-viable cells (34); iii) a wide variation between patients in antibody permeability for cells that show a similar dye permeability seems unlikely.

We investigated calreticulin exposure during in vivo treatment with ATRA, valproic acid and theophyllin. The apoptotic phenotype was then altered and cells collected after 2 days of ATRA therapy showed increased spontaneous in vitro exposure of calreticulin. These results suggest that altered regulation of apoptosis is observed after in vivo ATRA therapy in addition to altered regulation of angiogenesis, cell cycle progression and gene transcription (20).

HSP70 and HSP90 levels were significantly correlated after $18 \mathrm{~h}$ of in vitro culture, but these levels showed no correlation with calreticulin exposure. Thus, it seems likely that HSP70 and HSP90 release is at least partly dependent on the same regulatory mechanisms but with different kinetics, whereas HSP release and calreticulin exposure seem to be independent phenotypic characteristics during stress-induced apoptosis in primary human AML cells. The HSP levels do not simply reflect the degree of cell death because differences between 
patients pesisted in drug-containing cultures that generally showed a very low viability.

Our present results demonstrate that primary human AML cells can show functional characteristics consistent with immunogenic apoptosis. These characteristics show a wide variation between individual patients, but it is not known whether this biological heterogeneity contributes to the differences in leukemia relapse risk between patients.

\section{Acknowledgements}

The study was supported by the Norwegian Cancer Society and the Solveig and Ove Lunds Foundation.

\section{References}

1. Estey E and Dohner H: Acute myeloid leukaemia. Lancet 368: 1894-1907, 2006.

2. Oyan AM, Anensen N, Bo TH, et al: Genes of cell-cell interactions, chemotherapy detoxification and apoptosis are induced during chemotherapy of acute myeloid leukemia. BMC Cancer 9: 77,2009 .

3. Solary E, Droin N, Bettaieb A, Corcos L, Dimanche-Boitrel MT and Garrido C: Positive and negative regulation of apoptotic pathways by cytotoxic agents in hematological malignancies. Leukemia 14: 1833-1849, 2000.

4. Auletta JJ and Lazarus HM: Immune restoration following hematopoietic stem cell transplantation: an evolving target. Bone Marrow Transplant 35: 835-857, 2005.

5. Behl D, Porrata LF, Markovic SN, et al: Absolute lymphocyte count recovery after induction chemotherapy predicts superior survival in acute myelogenous leukemia. Leukemia 20: 29-34, 2006.

6. Kim DH, Sohn SK, Won DI, Lee NY, Suh JS and Lee KB: Rapid helper T-cell recovery above $200 \times 10$ 6/1 at 3 months correlates to successful transplant outcomes after allogeneic stem cell transplantation. Bone Marrow Transplant 37: 1119-1128, 2006.

7. Parkman R, Cohen G, Carter SL, et al: Successful immune reconstitution decreases leukemic relapse and improves survival in recipients of unrelated cord blood transplantation. Biol Blood Marrow Transplant 12: 919-927, 2006.

8. Porrata LF, Litzow MR, Tefferi A, et al: Early lymphocyte recovery is a predictive factor for prolonged survival after autologous hematopoietic stem cell transplantation for acute myelogenous leukemia. Leukemia 16: 1311-1318, 2002.

9. Bruserud O, Ersvaer E, Olsnes A and Gjertsen BT: Anticancer immunotherapy in combination with proapoptotic therapy. Curr Cancer Drug Targets 8: 666-675, 2008.

10. Obeid M, Tesniere A, Ghiringhelli F, et al: Calreticulin exposure dictates the immunogenicity of cancer cell death. Nat Med 13: 54-61, 2007.

11. Obeid M, Tesniere A, Panaretakis T, et al: Ecto-calreticulin in immunogenic chemotherapy. Immunol Rev 220: 22-34, 2007.

12. Tesniere A, Panaretakis T, Kepp O, et al: Molecular characteristics of immunogenic cancer cell death. Cell Death Differ 15: 3-12, 2008.

13. Michalak M, Groenendyk J, Szabo E, Gold LI and Opas M: Calreticulin, a multi-process calcium-buffering chaperone of the endoplasmic reticulum. Biochem J 417: 651-666, 2009.

14. Bernasconi P: Molecular pathways in myelodysplastic syndromes and acute myeloid leukemia: relationships and distinctions-a review. Br J Haematol 142: 695-708, 2008.

15. Schimmer AD: Novel therapies targeting the apoptosis pathway for the treatment of acute myeloid leukemia. Curr Treat Options Oncol 8: 277-286, 2007.

16. Hjelle SM, Forthun RB, Haaland I, et al: Clinical proteomics of myeloid leukemia. Genome Med 2: 41, 2010.
17. Liseth K, Ersvaer E, Hervig T and Bruserud O: Combination of intensive chemotherapy and anticancer vaccines in the treatment of human malignancies: the hematological experience. J Biomed Biotechnol 2010: 692097, 2010

18. Chaput N, De Botton S, Obeid M, et al: Molecular determinants of immunogenic cell death: surface exposure of calreticulin makes the difference. J Mol Med 85: 1069-1076, 2007.

19. Ryningen A, Stapnes C, Lassalle P, Corbascio M, Gjertsen BT and Bruserud O: A subset of patients with high-risk acute myelogenous leukemia shows improved peripheral blood cell counts when treated with the combination of valproic acid, theophylline and all-trans retinoic acid. Leuk Res 33: 779-787, 2009.

20. Ryningen A, Stapnes C, Paulsen K, Lassalle P, Gjertsen BT and Bruserud O: In vivo biological effects of ATRA in the treatment of AML. Expert Opin Investig Drugs 17: 1623-1633, 2008.

21. Bruserud O, Gjertsen BT, Foss B and Huang TS: New strategies in the treatment of acute myelogenous leukemia (AML): in vitro culture of aml cells-the present use in experimental studies and the possible importance for future therapeutic approaches. Stem Cells 19: 1-11, 2001.

22. Bruserud O: Effect of dipyridamole, theophyllamine and verapamil on spontaneous in vitro proliferation of myelogenous leukaemia cells. Acta Oncol 31: 53-58, 1992.

23. Bruserud O, Gjertsen BT and von Volkman HL: In vitro culture of human acute myelogenous leukemia (AML) cells in serumfree media: studies of native AML blasts and AML cell lines. J Hematother Stem Cell Res 9: 923-932, 2000.

24. Stapnes C, Ryningen A, Hatfield K, et al: Functional characteristics and gene expression profiles of primary acute myeloid leukaemia cells identify patient subgroups that differ in susceptibility to histone deacetylase inhibitors. Int J Oncol 31: 1529-1538, 2007.

25. Bruserud O, Hovland R, Wergeland L, Huang TS and Gjertsen BT: Flt3-mediated signaling in human acute myelogenous leukemia (AML) blasts: a functional characterization of Flt3-ligand effects in AML cell populations with and without genetic Flt3 abnormalities. Haematologica 88: 416-428, 2003.

26. Bruserud O, Huang TS, Glenjen N, Gjertsen BT and Foss B Leptin in human acute myelogenous leukemia: studies of in vivo levels and in vitro effects on native functional leukemia blasts. Haematologica 87: 584-595, 2002.

27. Ryningen A, Ersvaer E, Oyan AM, et al: Stress-induced in vitro apoptosis of native human acute myelogenous leukemia (AML) cells shows a wide variation between patients and is associated with low BCL-2:Bax ratio and low levels of heat shock protein 70 and 90. Leuk Res 30: 1531-1540, 2006.

28. Casares N, Pequignot MO, Tesniere A, et al: Caspase-dependent immunogenicity of doxorubicin-induced tumor cell death. J Exp Med 202: 1691-1701, 2005.

29. Weiden PL, Flournoy N, Thomas ED, et al: Antileukemic effect of graft-versus-host disease in human recipients of allogeneicmarrow grafts. N Engl J Med 300: 1068-1073, 1979.

30. Del Poeta G, Venditti A, Del Principe MI, et al: Amount of spontaneous apoptosis detected by $\mathrm{Bax} / \mathrm{Bcl}-2$ ratio predicts outcome in acute myeloid leukemia (AML). Blood 101: 2125-2131, 2003.

31. Reikvam H, Ersvaer E and Bruserud O: Heat shock protein $90-\mathrm{a}$ potential target in the treatment of human acute myelogenous leukemia. Curr Cancer Drug Targets 9: 761-776, 2009.

32. Bruserud O, Ryningen A, Wergeland L, Glenjen NI and Gjertsen BT: Osteoblasts increase proliferation and release of pro-angiogenic interleukin 8 by native human acute myelogenous leukemia blasts. Haematologica 89: 391-402, 2004.

33. Stapnes C, Doskeland AP, Hatfield K, et al: The proteasome inhibitors bortezomib and PR-171 have antiproliferative and proapoptotic effects on primary human acute myeloid leukaemia cells. Br J Haematol 136: 814-828, 2007.

34. Corver WE, Cornelisse CJ and Fleuren GJ: Simultaneous measurement of two cellular antigens and DNA using fluorescein-isothiocyanate, R-phycoerythrin, and propidium iodide on a standard FACScan. Cytometry 15: 117-128, 1994.

35. Mrozek K, Heerema NA and Bloomfield CD: Cytogenetics in acute leukemia. Blood Rev 18: 115-136, 2004. 